\title{
Accelerated Convergence to Steady State by Gradual Far-Field Damping
}

\author{
Smadar Karni* \\ University of Michigan, Ann Arbor, Michigan 48109
}

\begin{abstract}
Reflections from artificial boundaries inhibit convergence of transient solutions to their steady limit. Far-field damping operators to suppress such reflections are presented for general first-order hyperbolic systems, and particular reference is made to the compressible Euler equations. The damping operator has the following properties: 1) no reflections are generated due to the introduction of the damping terms and 2) different wave systems may be damped at different rates. Feature 1 is imperative for successful damping and enables the attenuation of waves over relatively short length scales. Feature 2 enables the damping operator to act selectively on the outgoing waves alone, leaving the incoming waves unharmed. This property is desirable in genuine time-dependent problems where consistent information should be allowed to propagate from the artificial boundaries into the domain. Results for compressible Euler flows past airfoils show the potential of far-field damping in substantially accelerating convergence, particularly in fully subsonic problems.
\end{abstract}

\section{Introduction}

$\mathbf{O}$ NE important factor that influences rapid convergence of computed transient solutions to steady state is the unsteadiness shed by the various boundary procedures. In particular, external flow calculations raise the problem of modeling open boundaries across which the fluid flows and which should ideally allow the outgoing disturbances to pass through without generating reflections. Apart from degrading time accuracy of transient solutions, reflected waves carry energies that bounce back and forth between artificial and solid boundaries dissipating very slowly ${ }^{6,15}$ and inhibiting convergence to the steady limit.

For evolutionary problems of hyperbolic character, perfectly nonreflecting boundary conditions (BCs) exist only in one space dimension. ${ }^{1-3}$ In multidimensional problems, perfectly absorbing $\mathrm{BCs}$ do not exist. ${ }^{3-5}$ Instead, one aims at minimizing the amount of reflected energy at the boundary, often by matching the BCs to a known (asymptotic) behavior of the solution in the far field ${ }^{1-10}$ or by applying heuristic boundary procedures guided by practical experience. ${ }^{11,12}$

In this paper, we pursue a less conventional approach to suppress far-field reflections based on modifying the governing equations in a narrow absorbing sponge layer near the far-field boundary. The far-field modification takes the form of a damping operator and is motivated by the natural decay of multidimensional disturbances that tend to zero strength as they approach infinity.

Variations on the theme of far-field damping were proposed before by various authors ${ }^{16-18}$ and applied to the solutions of external problems in various application areas. One universal feature of far-field damping is that the introduction of the damping terms creates an interface that may generate reflections when crossed by traveling waves. Damping operators that give rise to reflections are of limited potential. In particular, in order to reduce the effect of partial reflections, the damping operator has to be applied very gradually and smeared over a large region; inaccuracies in the converged

Received Dec. 3, 1990; presented as Paper 91-1604 at the AIAA 10th Computational Fluid Dynamics Conference, Honolulu, HI, June 24-27, 1991; revision received July 15, 1991; accepted for publication Aug. 9, 1991. Copyright (C) 1991 by the American Institute of Aeronautics and Astronautics, Inc. All rights reserved.

*Assistant Professor, Department of Mathematics. Member AIAA. solution are to be expected. In Refs. 13 and 14, it is shown that damping operators based on modified second-order scalar wavelike equations ${ }^{16-18}$ will inevitably give rise to partial reflections. In an aerodynamics context, this bears on potential flow descriptions [full potential, transonic small perturbations (TSP), etc.].

By contrast, the damping operator proposed in this paper is based on a modified first-order system of equations. First-order systems possess additional degrees of freedom and enable the construction of a damping operator with the following properties:

1) Traveling waves may be attenuated at an exponential rate.

2) Wave attenuation is reflection free, even under an abrupt change of coefficients.

3) Different wave systems may be damped at different rates.

4) The operator depends continuously on a set of damping functions; when all are set to zero, the unmodified equations (and solutions) are recovered.

By property 1 , the damping operator has a similar effect to moving the outer boundary to a very large distance, without incurring the penalty of large domains of computation. The question of which BCs to apply at the boundary itself is of much lesser importance since wave amplitudes near the boundary are very close to zero strength. Property 2 is imperative for an efficient attenuation process and for obtaining converged steady-state solutions that can be trusted. Property 3 enables the design of an operator that acts only on the outgoing part of the solution, leaving the incoming part unharmed. This feature is particularly desirable in genuinely time-dependent problems where time dependence enters through the far-field BCs and where consistent incoming information should be allowed to propagate into the domain. Property 4 makes the damping operator easy to implement. In an aerodynamics context, this is applicable to the Euler and Navier-Stokes flow models, as well as to the TSP equations written as a first-order system.

The outline of this paper is as follows. We construct farfield damping operators for general first-order hyperbolic systems in one space dimension, with particular reference to the compressible Euler equations, and analyze the resulting interface problem. We comment on the potential merits of combining the damping operator with other convergence accelerating devices such as multistage time integration, multigrids, and preconditioning. The damping technique is extended to two space dimensions where we also discuss damping 
strategies and wave reflection in two space dimensions as well as the discrete interface problem. We compare the present damping operator and the enthalpy damping device ${ }^{10,20}$ for accelerating convergence. We conclude by presenting numerical results of compressible Euler calculations, where far-field damping substantially accelerates convergence to steady state in both transonic and subsonic flow regimes, with a particularly dramatic impact in the fully subsonic case. We show that rapid convergence is maintained even when the outer boundaries are crudely overspecified to the freestream values, thus demonstrating the potential merit of the damping technique in handling near characteristic boundaries. In this case, transient inflow-outflow boundary type changes almost at random because of small numerical errors, and conventional boundary treatment via nonreflecting BCs may end up shedding unsteadiness and delay convergence.

\section{Equations}

\section{Analysis}

The one-dimensional Euler equations for inviscid non-heatconducting compressible flows in conservation form are

$$
\begin{gathered}
W_{t}+F(W)_{x}=0 \\
W=\left(\begin{array}{c}
\rho \\
\rho u \\
E
\end{array}\right), \quad F(W)=\left(\begin{array}{c}
\rho u \\
\rho u^{2}+p \\
\rho u H
\end{array}\right)
\end{gathered}
$$

where $\rho, u, p$, and $E$ are the density, velocity, pressure, and total energy, respectively, and $H=(E+p) / \rho$ is the specific enthalpy. We use $a$ to denote the speed of sound and $\gamma$ the specific heat ratio. Then by the perfect gas assumption, $p$ is calculated from

$$
p=(\gamma-1)\left(E-1 / 2 \rho u^{2}\right)
$$

We use $\boldsymbol{A}=\partial \boldsymbol{F} / \partial \boldsymbol{W}$ to denote the Jacobian matrix and use $\boldsymbol{R}=\left(r_{1}, r_{2}, r_{3}\right)$ and $\boldsymbol{\Lambda}=\operatorname{diag}\left(\lambda_{1}, \lambda_{2}, \lambda_{3}\right)$ to denote the matrices with the eigenvectors $r_{k}$ and eigenvalues $\lambda_{k}$ of $A$. The equations in quasilinear form are

$$
W_{t}+A(W) W_{x}=0
$$

$A$ is given by

$$
\boldsymbol{A}=\left[\begin{array}{ccc}
0 & 1 & 0 \\
\frac{\gamma-3}{2} u^{2} & (3-\gamma) u & \gamma-1 \\
-u\left(H-\frac{(\gamma-1)}{2} u^{2}\right) & H-(\gamma-1) u^{2} & \gamma u
\end{array}\right]
$$

and $\boldsymbol{R}$ and $\boldsymbol{\Gamma}$ are given by

$$
\begin{gathered}
\boldsymbol{R}=\left(\begin{array}{ccc}
1 & 1 & 1 \\
u-a & u & u+a \\
H-u a & 1 / 2 u^{2} & H+u a
\end{array}\right) \\
\Lambda=\left(\begin{array}{ccc}
u-a & 0 & 0 \\
0 & u & 0 \\
0 & 0 & u+a
\end{array}\right)
\end{gathered}
$$

Simple wave solutions to Eq. (1) are eigenvectors $\boldsymbol{r}_{k}$, moving at the corresponding speeds $\lambda_{k}$,
General single frequency solutions to Eq. (1) are superposition of simple waves

$$
W(x, t)=\sum_{k=1}^{3} \alpha_{k} e^{i \omega(t-x / \lambda k)} \boldsymbol{r}_{k}
$$

for some wave strengths $\alpha_{k}$.

\section{Far-Field Damping in One Dimension}

In the far field, we introduce the damping operator $D(W)$,

$$
W_{t}+A(W) W_{x}+D(W)\left(W-W_{\infty}\right)=0
$$

where $D(W)$ is the $3 \times 3$ matrix

$$
\begin{gathered}
\boldsymbol{D}(W)=\boldsymbol{R}(W) \Delta(W) R^{-1}(W) \\
\Delta(W)=\operatorname{diag}\left(d_{k} \lambda_{k}\right)
\end{gathered}
$$

where $\boldsymbol{R}(\boldsymbol{W})$ and $\lambda_{\mathrm{k}}$ are the eigenvectors and eigenvalues of $\boldsymbol{A}$ given by Eqs. (4), and $d_{k}$ are arbitrary damping coefficients. General single frequency solutions to Eq. (6) are

$$
W(x, t)=\sum_{k=1}^{3} \alpha_{k} e^{-d_{k} x} e^{i \omega(t-x / \lambda k)} \boldsymbol{r}_{k}
$$

representing plane wave solutions $\boldsymbol{r}_{k}$, moving at speeds $\lambda_{k}$, and exponentially attenuated at rates $d_{k}$. The choice $d_{k}=0$ recovers the undamped solution for the $k$ th wave. Setting all $d_{k}$ to zero recovers the unmodified solution (5).

An attractive form of the damping operator that does not involve expensive matrix products can be obtained by decomposing the far-field perturbation $\boldsymbol{W}-\boldsymbol{W}_{\infty}$ into eigenmodes

$$
\boldsymbol{W}-\boldsymbol{W}_{\infty}=\sum_{k=1}^{3} \alpha_{k} \boldsymbol{r}_{k}
$$

Then,

$$
\boldsymbol{D}(\boldsymbol{W})\left(\boldsymbol{W}-\boldsymbol{W}_{\infty}\right)=\sum_{k=1}^{3} \alpha_{k} \lambda_{k} d_{k} \boldsymbol{r}_{k}
$$

where $d_{k}$ are the damping coefficients. The $\alpha_{k}$ are given by the usual expressions of the Roe's solver ${ }^{19}$

$$
\begin{gathered}
\alpha_{1}=\frac{\Delta p-\rho a \Delta u}{2 a^{2}} \\
\alpha_{2}=\frac{\Delta \rho-a^{2} \Delta p}{a^{2}} \\
\alpha_{3}=\frac{\Delta p+\rho a \Delta u}{2 a^{2}}
\end{gathered}
$$

where $\Delta()=()-()_{\infty}$ is the local perturbation of the respective flow variables about their freestream values.

\section{Reflection Analysis}

The introduction of the damping terms in the governing equations creates an interface to either side of which different equations are being solved and across which wave motion is to remain continuous. To analyze reflection and transmission properties of the damping operator, we consider the problem

$$
\begin{array}{ll}
W_{t}+A(W) W_{x}+D_{1}(W)\left(W-W_{\infty}\right)=0 & x<0 \\
W_{t}+A(W) W_{x}+D_{2}(W)\left(W-W_{\infty}\right)=0 & x>0
\end{array}
$$

$$
\boldsymbol{W}(x, t)=e^{i \omega(t-x / \lambda k)} \boldsymbol{r}_{k}
$$$$
[\boldsymbol{W}]_{x=0}=0
$$ 
where $[W]$ denotes the jump in $W$ across the interface. $D_{1}$ and $D_{2}$ are possibly different damping matrices of the general form

$$
\begin{gathered}
D_{1}(W)=R(W) \Delta_{1}(W) R^{-1}(W) \\
D_{2}(W)=R(W) \Delta_{2}(W) R^{-1}(W) \\
\Delta_{1}(W)=\operatorname{diag}\left(d_{k}^{-} \lambda_{k}\right) \\
\Delta_{2}(W)=\operatorname{diag}\left(d_{k}^{+} \lambda_{k}\right)
\end{gathered}
$$

where $d_{k}^{ \pm}$are arbitrary damping coefficients to either side of the interface $x=0$. By Eq. (8), respective solutions take the form

$$
\begin{array}{ll}
W(x, t)=\sum_{k=1}^{3} \alpha_{k}^{-} e^{-d_{k} x} e^{i \omega(t-x / \lambda k)} \boldsymbol{r}_{k} & x<0 \\
W(x, t)=\sum_{k=1}^{3} \alpha_{k}^{+} e^{-d_{k}^{+} x} e^{i \omega(t-x / \lambda k)} \boldsymbol{r}_{k} & x>0
\end{array}
$$

Continuity across the interface reduces to

$$
\boldsymbol{W}(0, t)=\sum_{k=1}^{3} \alpha_{k}^{-} \boldsymbol{r}_{k}(\boldsymbol{W})=\sum_{k=1}^{3} \alpha_{k}^{+} r_{k}(\boldsymbol{W})
$$

The eigenvectors $r_{k}$ are independent; hence, they form a basis with respect to which the representation of $W(0, t)$ is unique. It then follows that $\alpha_{k}^{ \pm}$satisfy

$$
\alpha_{k}^{-}=\alpha_{k}^{+} \quad \forall t>0
$$

implying full transmission of all waves at all times $t>0$. We summarize the main properties of the far-field damping operator:

1) Analytic solution is fully transmitted across the interface for arbitrarily large damping coefficients.

2) Different wave systems may be attenuated at different rates, and in particular, the outgoing waves may be damped while the incoming waves are left unharmed.

In both of these respects, the present damping operator represents an improvement over damping operators previously proposed. ${ }^{16-18}$ The latter are all based on a modified secondorder scalar wavelike equation, whereas the present operator is based on a modified first-order system. A simple count of degrees of freedom confirms that, if the second order wave equation is modified, there are only two free coefficients to specify in order to determine the decay rates of all of the wave systems, whereas if the same equation is transformed into the derivative space and written as a $2 \times 2$ first-order system, there are four matrix elements to determine the decay rates. The additional freedom is also reflected by the fact that every second-order scalar equation can be written as a first-order system, but not every first order system can be reduced to a second-order scalar. It can also be verified that preservation of the eigenvectors, which is imperative for full transmission of waves, is not possible with a damped second-order scalar wave equation. ${ }^{13,14}$

\section{Far-Field Damping and Other Accelerating Devices}

The damping operator is derived on the continuous level, without reference to any particular numerical scheme. Because of its generality, the far-field damping can be applied to any numerical algorithm and can be used easily in conjunction with other convergence accelerating devices such as multiple grids, multistage time integration, and preconditioning. Note, however, that if the system is preconditioned, the damping terms should be preconditioned in the same way in order to retain its nonreflective features. Note also that, in multiple grid algorithms, the amount of extra computational burden incurred by the coarse and intermediate grid levels is negligible since the region in which the equations are modified is very narrow.

\section{Far-Field Damping in Two Dimensions}

The two-dimensional Euler equations in conservation form are

$$
\boldsymbol{W}_{t}+\boldsymbol{F}(\boldsymbol{W})_{x}+\boldsymbol{G}(\boldsymbol{W})_{y}=0
$$

where

$$
W=\left[\begin{array}{c}
\rho \\
\rho u \\
\rho v \\
E
\end{array}\right], \quad F(W)=\left[\begin{array}{c}
\rho \\
\rho u^{2}+p \\
\rho u v \\
\rho u H
\end{array}\right], \quad F(W)=\left[\begin{array}{c}
\rho v \\
\rho u v \\
\rho v^{2}+p \\
\rho v H
\end{array}\right]
$$

In quasilinear form, the equations are

$$
W_{t}+A(W) W_{x}+B(W) W_{y}=0
$$

where $A$ and $B$ are the Jacobian matrices $A=\partial F / \partial W$ and $\boldsymbol{B}=\partial \boldsymbol{G} / \partial \boldsymbol{W}$ and are given by

$$
\begin{aligned}
& A=\left[\begin{array}{cccc}
0 & 1 & 0 & 0 \\
\frac{\gamma-1}{2} q^{2}-u^{2} & (3-\gamma) u & -(\gamma-1) v & \gamma-1 \\
-u v & v & u & 0 \\
-u\left(H-\frac{\gamma-1}{2} q^{2}\right) & H-(\gamma-1) u^{2} & -(\gamma-1) u v & \gamma u
\end{array}\right] \\
& \boldsymbol{B}=\left[\begin{array}{cccc}
0 & 0 & 1 & 0 \\
-u v & v & u & 0 \\
\frac{\gamma-1}{2} q^{2}-v^{2} & -(\gamma-1) u & (3-\gamma) v & \gamma-1 \\
-v\left(H-\frac{\gamma-1}{2} q^{2}\right) & -(\gamma-1) u v & H-(\gamma-1) v^{2} & \gamma^{v}
\end{array}\right]
\end{aligned}
$$


where $|q|=\sqrt{\left(u^{2}+v^{2}\right)}$ is the flow velocity. Plane wave solutions in an arbitrary direction $\theta$ satisfy

$$
W_{t}+M(\theta) W_{x^{\prime}}=0
$$

where $x^{\prime}=x \cos \theta+y \sin \theta$, and $M(\theta)=A(W) \cos \theta+B(W)$ $\sin \theta$. The right eigenvector matrix of $M(\theta)$ is

$$
\boldsymbol{R}(\theta)=\left[\begin{array}{cccc}
1 & 1 & 0 & 1 \\
q_{n}-a & q_{n} & 0 & q_{n}+a \\
q_{t} & q_{t} & 1 & q_{t} \\
H-q_{n} a & 1 / 2 q^{2} & q_{t} & H+q_{n} a
\end{array}\right]
$$

where $q_{n}=u \cos \theta+v \sin \theta, q_{t}=-u \sin \theta+v \cos \theta$ are the velocity components parallel and normal to the direction $\theta$. By previous analysis, we construct a $\theta$ dependent damping operator

$$
W_{t}+F(W)_{x}+G(W)_{y}+D(\theta)\left(W-W_{\infty}\right)=0
$$

with

$$
\begin{gathered}
\boldsymbol{D}(\theta)=\boldsymbol{R}(\theta) \dot{\Delta}(\theta) \boldsymbol{R}^{-1}(\theta) \\
\Delta(\theta)=\operatorname{diag}\left[d_{k} \lambda_{k}(\theta)\right]
\end{gathered}
$$

Equation (14) preserves the eigenvectors in an arbitrary direction $\theta$, and has plane wave solutions

$$
W(x, t)=\sum_{k} \alpha_{k} e^{-d_{k}} e^{i \omega\left(t-x^{\prime} / \lambda k\right)} r_{k}
$$

describing plane waves moving at an arbitrary direction $x^{\prime}$ and decaying at exponential rates $d_{k}$. If all wave systems are damped at the same rate, $d_{k} \equiv d$ for all $k$, and Eq. (14) reduces to

$$
W_{t}+F(W)_{x}+G(W)_{y}+d M(\theta)\left(W-W_{\infty}\right)=0
$$

It is, however, not necessary to constrain all wave systems by the same decay rate and the damping operator may remain in its general form (14). Here again a more attractive form to compute the damping operator is

$$
\boldsymbol{D}(\theta)\left(W-W_{\infty}\right)=\sum_{k} \alpha_{k} \lambda_{k} d_{k} r_{k}(\theta)
$$

with $\alpha_{k}$ the standard Roe's wave strengths.

\section{Wave Reflection in Two Dimensions}

Once the damping angle $\theta$ has been selected at each gridpoint, waves traveling locally in the designed angle are attenuated without any reflections. However, waves traveling at angles $\theta^{\prime}$ close to the designed angle $\theta$ do experience weak reflections since eigenvectors in the direction $\theta^{\prime}$ are not strictly

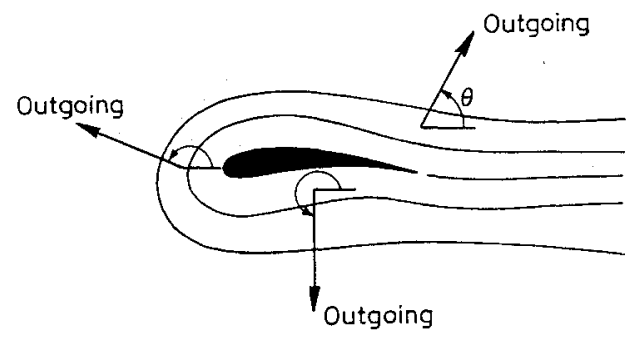

Fig. 1 Choosing the damping angle $\theta$ in the grid normal direction for airfoil calculations on C-grids.

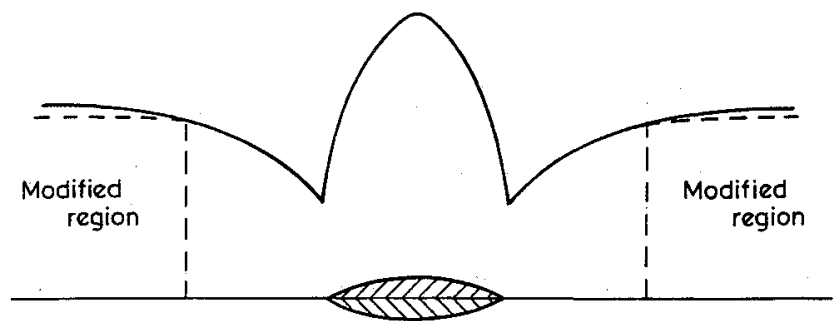

Fig. 2 Typical effect of far-field damping on converged subsonic Mach number profiles: undamped (dashed) and damped (solid).

preserved. To see that wave reflections are weak, we observe that a plane wave solution in a direction $\theta^{\prime}$ can be expressed as a sum of simple waves in the designed angle $\theta$

$$
r_{l}\left(\theta^{\prime}\right)=\sum_{k} \alpha_{k} r_{k}(\theta)
$$

where $\alpha_{k}$ are the wave strengths for this particular representation. It is easy to verify that for $|\Delta \theta|=\left|\theta-\theta^{\prime}\right| \ll 1, \alpha_{i} \sim 1$ and the rest of the $\alpha_{k}$ are of the order $|\Delta \theta|$ and are much less than 1. For example, consider a subsonic outflow boundary at some positive $x$ value. Damping in grid normal direction implies $\theta=0$. For an outgoing acoustic wave traveling at some small but nonzero angle $\theta^{\prime}$,

$$
r_{4}\left(\theta^{\prime}\right)=\sum_{k=1}^{4} \alpha_{k} r_{k}(0)
$$

and a simple calculation shows that

$$
\begin{array}{cc}
\alpha_{1}=v \theta^{\prime}(\gamma-2) / 2 a, & \alpha_{2}=-v \theta^{\prime}(\gamma-1) / a \\
\alpha_{3}=-u \theta^{\prime}, & \alpha_{4}=1+v \gamma \theta^{\prime} / 2 a
\end{array}
$$

In this case, the only incoming wave system is the first one, and we see that $\alpha_{1}$ is of the order of $\theta^{\prime}$ and is further multiplied by $v$, which is a small quantity. In many applications, far-field wave propagation is characterized by a clear dominant direction of propagation. ${ }^{15}$ In such cases, the success of far-field damping rests upon identifying and selecting an appropriate damping angle $\theta$. Finally, we observe that the damping terms do not affect incoming waves in the designed angle $\theta$, and have only a small effect on waves propagating at angles close to $\theta$. Thus, it allows faithful propagation of incoming information in directions close to $\theta$. In applications where the incoming wave angle $\theta^{\prime}$ is very different from the outgoing wave angle $\theta$, the present far-field damping is probably not suitable.

\section{Damping Strategies}

At each grid point, an angle $\theta$ has to be specified in order to select the direction of wave attenuation. Here, various damping strategies may be adopted. One strategy is the damping of the waves in the grid normal direction. In this case, $\theta=\theta_{i, j}$ is calculated and stored once at the beginning of the computation. A second strategy is the damping of the waves in the outgoing flow direction. This direction may be extracted from local flow gradients. Strictly, this direction needs to be computed at every time step. However, since far-field wave propagation has a very repetitive pattern that emerges right from very early stages of the calculation, ${ }^{15}$ the outgoing flow directions may remain at some frozen values $\theta_{i, j}$. This is probably a better strategy, particularly since, in contrast to common belief, far-field wave propagation is highly nonaligned with the grid, especially in calculations that employ local time stepping integration. ${ }^{15}$ The first strategy is illustrated in Fig. 1. The expected effect of the damping on far-field solutions is to pull the solution toward the freestream values. In the inner, unmodified region, solutions obtained with and without damping are expected to coincide. Inside the damping layer, 
Table 1 Converged values of $C_{N}$ and $C_{D}$ vs boundary distance

\begin{tabular}{lccccc}
\hline \hline $\begin{array}{l}\text { Test } \\
\text { number }\end{array}$ & $\begin{array}{c}\text { Boundary } \\
\text { distance }\end{array}$ & $\begin{array}{c}\text { Grid } \\
\text { size }\end{array}$ & $C_{N}$ & $C_{D}$ & $\begin{array}{c}\text { Number of } \\
\text { iterations }\end{array}$ \\
\hline \multicolumn{5}{c}{$M_{\infty}=0.5$} \\
1 & 1.00000 & $65 \times 17$ & -0.281787 & 0.001360 & 5390 \\
2 & 2.64355 & $85 \times 27$ & -0.313781 & 0.000478 & 5168 \\
3 & 4.70732 & $97 \times 33$ & -0.317904 & 0.000363 & 6358 \\
4 & 6.90649 & $105 \times 37$ & -0.318964 & 0.000353 & 6503 \\
5 & 10.12629 & $113 \times 41$ & -0.319406 & 0.000358 & 6418 \\
6 & 12.25938 & $117 \times 43$ & -0.319529 & 0.000342 & 6289 \\
\multicolumn{5}{c}{} \\
7 & 1.00000 & $65 \times 17$ & -0.355310 & 0.005332 & 1637 \\
8 & 2.64355 & $85 \times 27$ & -0.437400 & 0.012209 & 3919 \\
9 & 4.70732 & $97 \times 33$ & -0.450744 & 0.013626 & 4540 \\
10 & 6.90649 & $105 \times 37$ & -0.460982 & 0.014580 & 5342 \\
11 & 10.12629 & $113 \times 41$ & -0.462811 & 0.014788 & 5528 \\
12 & 12.25938 & $117 \times 43$ & -0.463335 & 0.014851 & 5879 \\
\hline \hline
\end{tabular}

the two solutions are expected to be different, the damped solution having converged values closer to the freestream values. This is illustrated in Fig. 2.

\section{Discrete Far-Field Damping}

Analytically, plane waves in an arbitrary direction $\theta$ may be wiped out by an abrupt change of damping coefficients without generating reflections. On the discrete level, the interface created due to the introduction of the damping terms is no longer a single point. It transforms into an interface zone, the width of which depends on that of the numerical stencil, that is on the order of accuracy of the numerical scheme. Since high-order schemes possess additional spurious solution modes, the damping operator has to be applied gradually in space in order to ensure that the damping procedure is numerically stable. In two space dimensions, even in cases where wave propagation in the far field is characterized by a single dominant direction of propagation, waves propagating at angles close to $\theta$ do experience weak reflections. To reduce the effect of these reflections and to enhance numerical stability, damping is applied gradually in space, yielding a damping layer. Yet the analytic nonreflective properties of the damping operator allow the width of the absorbing layer to be kept to a minimum, typically no more than four to six grid points across the modified region.

\section{Far-Field Damping and Enthalpy Damping}

Enthalpy damping, originally proposed by Jameson et al., ${ }^{10}$ proved a very successful device for accelerating convergence of unsteady compressible inviscid flows to their steady limit. It was derived by reducing the unsteady Euler equations to the wave equation, modifying the wave equation by adding a damping term to obtain decaying solutions as $t \rightarrow \infty$, and transforming the modified equation back to the Euler equations. The result is a set of modified equations

$$
W_{t}+\boldsymbol{F}(W)_{x}+\boldsymbol{G}(\boldsymbol{W})_{y}+d\left(\boldsymbol{H}-\boldsymbol{H}_{\infty}\right) \tilde{W}=0
$$

where $\boldsymbol{H}_{\infty}$ is the freestream enthalpy. Since the steady-state enthalpy is constant along streamlines, if all streamlines originate from a uniform freestream, steady solutions of Eq. (16) are also steady solutions of Eq. (11). The coefficient $d$ is then chosen to optimize convergence rate. Enthalpy damping was later analyzed by Jespersen, ${ }^{20}$ who made the following observations: When $d=0$, Eq. (16) forms a hyperbolic system, and possesses frozen coefficient solutions of the form

$$
W(x, t)=e^{-i \xi x^{\prime}} \sum_{k} \alpha_{k} e^{\omega k} r_{k}(\theta)
$$

with $\omega_{k}=\omega_{k}(\xi)$ purely imaginary. He also showed that under certain conditions, taking $d>0$ pulls $\omega_{k}$ off of the imaginary axis into the left half of the complex plane, yielding exponential time decay of solutions and, consequently, accelerated convergence. In fact, what he showed was that, out of the four eigenvalues $\omega_{k}(\xi)$, only three are pulled off while one always remains on the imaginary axis. Hence, even with enthalpy damping, not all solutions decay in time. He accredited the fact that Euler solutions do converge to a steady limit to the numerical dissipation introduced by the stable time integration procedure.

It is easy to verify that the present damping operator pulls all eigenvalues off of the imaginary axis. Moreover, they can be pulled as far off of the imaginary axis as one wishes by choosing appropriate values for the damping coefficients $d_{k}$. Enthalpy damped solutions are not time accurate, but they converge to the correct steady limit. By contrast, the present modified system (14) does not possess the same steady solutions as the original system (11). On the contrary, one expects far-field solutions to decay faster toward their ( $)_{\infty}$ values. On the other hand, since the damping terms are nonreflective, at least in a one-dimensional asymptotic sense, their introduction in Eq. (14) should not destroy time accuracy in the inner unmodified region and is expected to converge to the same steady limit over that region (see Fig. 2).

\section{Numerical Results}

Numerical Algorithm and Boundary Conditions

\section{Numerical Scheme}

The method of solution is Hall's variation ${ }^{11}$ of the cell vertex scheme with Lax-Wendroff (LW) time integration, originally proposed by $\mathrm{Ni} .{ }^{12}$ The time increment at cell vertex 1 (see Fig. 3) is given by

$$
\begin{gathered}
\delta W_{1}=\Delta_{1} W+\Delta_{2} W \\
\Delta_{1} W=\frac{S_{A} \Delta W_{A}+S_{B} \Delta W_{B}+S_{C} \Delta W_{C}+S_{D} \Delta W_{D}}{S_{A}+S_{B}+S_{C}+S_{D}} \\
\Delta_{2} W=\left[\left(\Delta F_{C}-\Delta F_{A}\right)\left(y_{B}-y_{D}\right)\right. \\
+\left(\Delta F_{D}-\Delta F_{B}\right)\left(y_{C}-y_{A}\right)+\left(\Delta G_{A}-\Delta G_{C}\right)\left(x_{B}-x_{D}\right) \\
\left.+\left(\Delta G_{B}-\Delta G_{D}\right)\left(x_{C}-x_{A}\right)\right] \times \frac{\Delta t}{4 S_{1}}
\end{gathered}
$$

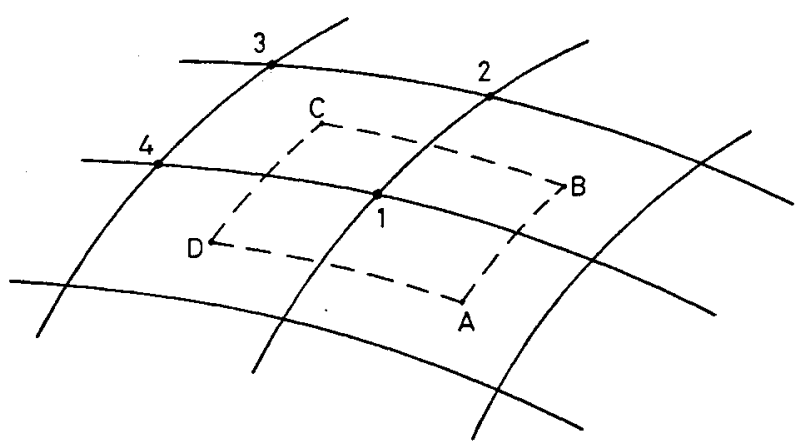

Fig. 3 Grid notation for Hall's numerical scheme given by Eqs. (17).

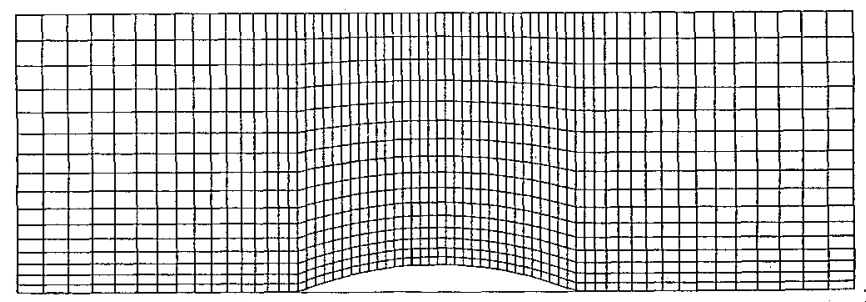

Fig. 4 Numerical grid for symmetric airfoil calculations. 
Table 2 Parameter study for far-field damping-transonic case

\begin{tabular}{lcccccc}
\hline \hline $\begin{array}{l}\text { Test } \\
\text { number }\end{array}$ & $\begin{array}{c}\text { Damping } \\
\text { coefficients }\end{array}$ & $\begin{array}{c}\text { Boundary } \\
\text { thickness }\end{array}$ & $\begin{array}{c}\text { Direction } \\
\text { of modification }\end{array}$ & $\begin{array}{c}\text { Number of } \\
\text { iterations }\end{array}$ & $C_{N}$ & $C_{D}$ \\
\hline 1 & 0.0 & 0 & - & 5528 & -0.46281 & 0.01479 \\
2 & 0.5 & 6 & $X$ & 5368 & -0.46443 & 0.01515 \\
3 & 0.5 & 6 & $Y$ & 4799 & -0.46116 & 0.01462 \\
4 & 0.4 & 5 & $Y$ & 4926 & -0.46158 & 0.01466 \\
5 & 0.6 & 5 & $Y$ & 4884 & -0.46144 & 0.01465 \\
6 & 0.5 & 6 & $X-Y$ & 3621 & -0.46355 & 0.01510 \\
7 & 0.4 & 6 & $X-Y$ & 3646 & -0.46355 & 0.01510 \\
8 & 0.5 & 6 & $X-Y$ & 3712 & -0.46372 & 0.01510 \\
\hline \hline
\end{tabular}

Table 3 Parameter study for far-field damping-subsonic case

\begin{tabular}{lcccccc}
\hline $\begin{array}{l}\text { Test } \\
\text { number }\end{array}$ & $\begin{array}{c}\text { Damping } \\
\text { coefficients }\end{array}$ & $\begin{array}{c}\text { Boundary } \\
\text { thickness }\end{array}$ & $\begin{array}{c}\text { Direction } \\
\text { of modification }\end{array}$ & $\begin{array}{c}\text { Number of } \\
\text { iterations }\end{array}$ & $C_{N}$ & $C_{D}$ \\
\hline 1 & 0.0 & 0 & - & 6418 & -0.31941 & 0.00036 \\
2 & 0.5 & 6 & $X$ & 5573 & -0.31976 & 0.00036 \\
3 & 0.5 & 6 & $X-Y$ & 2404 & -0.32007 & 0.00036 \\
4 & 0.4 & 6 & $X-Y$ & 2418 & -0.23005 & 0.00036 \\
5 & 0.5 & 6 & $Y$ & 3192 & -0.31985 & 0.00036 \\
6 & 0.4 & 5 & $Y$ & 3383 & -0.31995 & 0.00036 \\
7 & 0.5 & 5 & $Y$ & 3352 & -0.31997 & 0.00037 \\
8 & 0.5 & 5 & $X-Y$ & 2401 & -0.32001 & 0.00036 \\
\hline \hline
\end{tabular}

where $S_{A}$ denotes the area of cell $A$, and $\Delta t$ denotes the time step. The changes at cell centers are given by

$$
\begin{aligned}
& \Delta W_{C}=\left[\left(F_{3}-F_{1}\right)\left(y_{2}-y_{4}\right)+\left(F_{4}-F_{2}\right)\left(y_{3}-y_{1}\right)\right. \\
&+\left.\left(G_{1}-G_{3}\right)\left(x_{2}-x_{4}\right)+\left(G_{2}-G_{4}\right)\left(x_{3}-x_{1}\right)\right] \times \frac{\Delta t}{2 S_{C}} \\
& \Delta F_{C}=\left(\frac{\partial F}{\partial W}\right)_{C} \Delta W_{C}=\frac{1}{4}\left(A_{1}+A_{2}+A_{3}+A_{4}\right) \Delta W_{C} \\
& \Delta G_{C}=\left(\frac{\partial G}{\partial W}\right)_{C} \Delta W_{C}=\frac{1}{4}\left(B_{1}+B_{2}+B_{3}+B_{4}\right) \Delta W_{C}
\end{aligned}
$$

where $A_{1}$ and $B_{1}$ denote the Jacobian matrices at cell vertices. The scheme also uses artificial viscosity to suppress the spatial oscillations associated with symmetric schemes such as the LW scheme, the details of which can be found in Ref. 11 .

\section{Boundary Conditions}

The original scheme proposed by Hall ${ }^{11}$ is designed for the calculation of steady solutions. The energy equation is omitted and pressure is calculated from Bernoulli's equation, using freestream enthalpy. The boundary procedures recommended by Hall are the following. At inflow, tangential velocity and entropy are specified and normal velocity is extrapolated using a one-sided version of the interior scheme. At outflow, freestream pressure is specified and velocity components are extrapolated using a one-sided version of the interior scheme. These boundary procedures were selected by Hall after extensive experiments with various $\mathrm{BCs}$, including nonreflective boundary conditions. They have proved better suited for the calculation of steady solutions, in that they yielded faster convergence than the nonreflective BCs. Since the purpose of the present study is to test the damping technique rather than the BCs, the recommended BCs are used. On the solid wall, the interior scheme is applied and a simple tangency condition is applied to the predicted solution. In the tests presented in this paper, Hall's method is applied to the full $4 \times 4$ system (11). Hall's boundary procedures are augmented by specifying the freestream enthalpy at inflow and extrapolating enthalpy at outflow, using the same one-sided version of the interior scheme. It is the experience of the author that, like in the reduced system case, these boundary procedures yield faster convergence than nonreflecting boundary conditions and that the spatial decay of converged flowfield variables toward the freestream values is monotone.

\section{Damping Term}

The nonhomogenous system (14) is solved by an operator split algorithm where in the first stage the solution $W$ gets updated by the flux terms and in the second stage a pointwise time integrator is used to account for the effects of the damping operator. Since in our tests time accuracy is not required inside the modified layer in the far field, a first-order Euler time integrator is used. In cases where time accuracy is required, a more accurate time-integration scheme should be used.

\section{Numerical Tests}

Steady solutions are calculated for symmetric transonic and subsonic flows past a double circular airfoil of $10 \%$ height/ chord ratio. The unsteady compressible Euler equations (11) are integrated and convergence is declared when nodal changes of all conserved quantities are less than $10^{-6}$. We use an aligned mesh with 32 evenly spaced cells on the airfoil and linearly stretched cells on either of its sides and in the vertical direction (see Fig. 4). Converged steady-state drag and normal force coefficients are obtained using

$$
\begin{aligned}
& C_{D}=\sum_{i} \frac{\left(p_{i}-p_{\infty}\right) \Delta y_{i}}{\rho_{\infty} u_{\infty}^{2}} \\
& C_{N}=\sum_{i} \frac{\left(p_{i}-p_{\infty}\right) \Delta x_{i}}{\rho_{\infty} u_{\infty}^{2}}
\end{aligned}
$$

Owing to symmetry, the solution is calculated only on the top half of the airfoil, with reflective BCs applied along the line of symmetry. Consequently, the normal force coefficient $C_{N}$ does not vanish and serves to assess the effect of the far-field boundary and the far-field damping on the accuracy of the converged solution.

\section{Boundary Effect Accuracy Test}

To minimize the effect of the far-field BCs on the converged solution, we conducted a series of experiments with the outer boundary placed at various increasingly larger distances. The effect of the far-field boundaries is considered eliminated 
when moving the outer boundary farther out contributes only negligible changes to the converged solution (changes in $C_{N}$ and $C_{D} \leq 10^{-4}$ ). The results of the transonic and subsonic flow calculations are summarized in Table 1. For the tests conducted, it was found that a boundary distance of $\sim 10$ chord lengths is sufficient and solutions of tests 5 and 11 are considered accurate. The respective converged Mach number and pressure profiles are shown in Fig. 5.

\section{Far-Field Damping Effect}

The effect of far-field damping on the convergence characteristics of the solution is summarized in Tables 2 and 3 . In all of the following tests, damping is applied in the grid normal direction, with acoustic, shear, and entropy outgoing waves all damped at the same rate. In the tables, boundary thickness indicates the number of nodes across the modified region, direction of modification indicates the grid normal direction in which damping is applied ( $X$ for inflow and outflow boundaries, $Y$ for top boundary, and $X-Y$ for both), and damping coefficient indicates the choice of $d_{k}$. The first line in each table serves as a benchmark and indicates a computation with the BCs described in the previous section. Tests 2-7 use the same BCs together with far-field damping. In test 8 , all outer boundaries are crudely overspecified to freestream conditions, and only far-field damping is applied. The purpose of test 8 is not to advocate overspecification of artificial boundaries. We do believe that the boundary type should be correctly identified. This, however, is not always easy. For example, near characteristic boundaries may change their type almost at random during the transient phase of the calculation. In this case, a conventional boundary treatment that depends on the boundary type will also be random and may end up shedding unsteadiness and delaying convergence. One way to avoid this is to (over)specify freestream conditions on the boundary and use far-field damping to damp the solution towards the specified state.

\section{Transonic Test Case}

Results are summarized in Table 2. In all cases, far-field damping accelerates convergence to steady states. The value a)
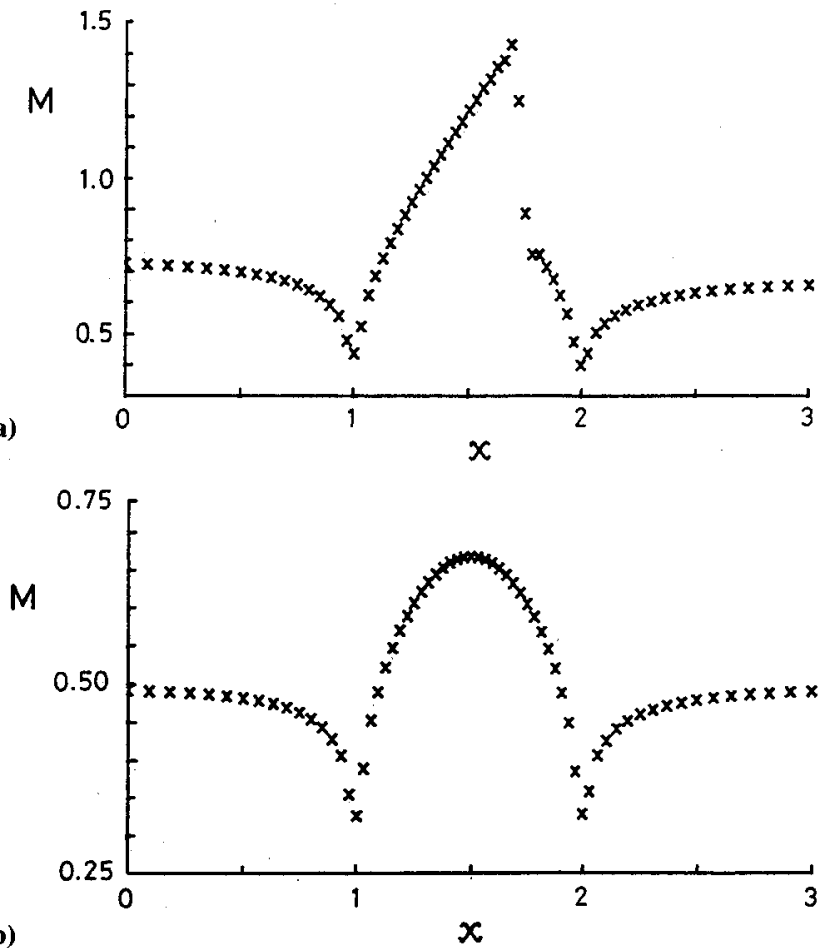

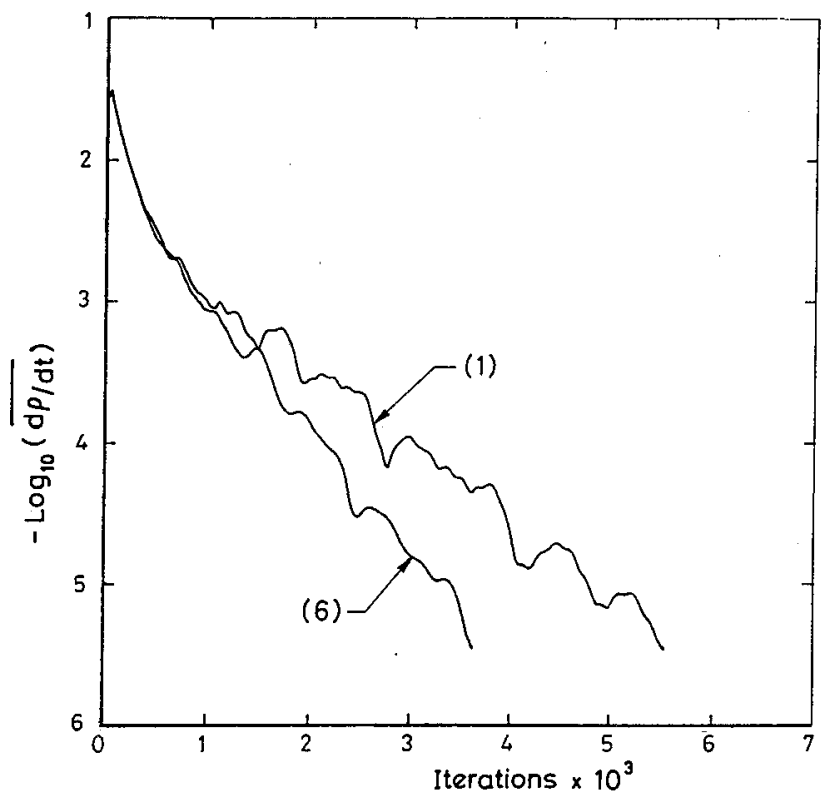

Fig. 6 Density residual history for transonic test case for tests 1 and 6 in Table 2.

Fig. 7 Density residual history for subsonic test case for tests 1 and 3 in Table 3.

$d_{k} \sim 0.5$ seems like a good choice, yielding fast convergence. Within the range tested, results are not very sensitive to the choice of attenuation rate. What seems to matter more is the thickness of the boundary, or more precisely, the distance of the damping layer from the airfoil. This is to be expected since far-field damping requires that the local flowfield is close to linear, a condition that is not met close to the airfoil. The appropriate distance of the boundary layer from the airfoil is problem dependent and depends on the extent of the disturbed flowfield away from the airfoil. For the tests conducted, the boundary layer distance is $\sim 7$ chords with four to six nodes across its width. Optimal values for other problems may be different. Comparison of tests 1 and 6 reveals a 35\% drop in the number of iterations required to reach steady state, with relative differences in $C_{N}$ and $C_{D}$ less than $1 \%$. Absolute differences in both are of order $10^{-4}$. Convergence histories of tests 1 and 6 are compared in Fig. 6. One striking result is that even the overspecified case 8 converges almost as rapidly

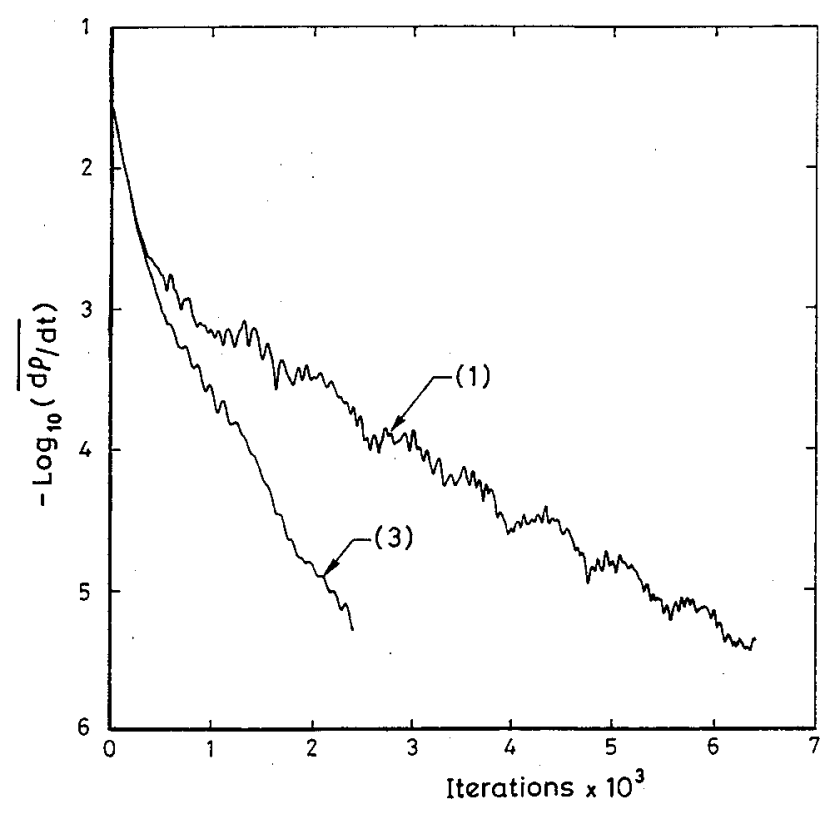

Fig. 5 Converged Mach number profiles for tests 5 and 11 in Table 1: a) $M_{\infty}=0.75$; b) $M_{\infty}=0.5$. 
as case 6 . This indicates that, in all previous cases, most energy is absorbed by the damping operator and not by the BCs.

\section{Subsonic Test Case}

Results are sumarized in Table 3 . Here, the impact of farfield damping is even more dramatic, yielding an impressive drop of over $60 \%$ in number of iterations required to reach steady state, with relative changes in $C_{N}$ and $C_{D}$ well under $0.5 \%$ (compare tests 1 and 3 ). The more pronounced impact of the damping is to be expected, since in the transonic case, another source of unsteadiness is the shock formation and its slow convergence to the steady shock profile. The rapid convergence of test 8 should also be noted, demonstrating the effectiveness of energy absorption by the damping operator even when the boundary is overspecified. Convergence histories of tests 1 and 3 show that the periodic nature of convergence, attributable to far-field reflections, ${ }^{15}$ is almost entirely removed (see Fig. 7).

\section{Summary}

A framework has been given for the construction of damping operators to suppress reflections from artificial boundaries for general first-order hyperbolic systems. Particular reference is made to the compressible Euler equations. The damping operator selectively damps the outgoing part of the solution over short length scales, without affecting the incoming part of the solution and without generating reflections. Two-dimensional steady Euler calculations of subsonic and transonic flows past symmetric airfoils demonstrate substantial improvement in convergence characteristics, with a particularly sharp impact in the fully subsonic case. The results also indicate that far-field damping is an attractive alternative to conventional boundary treatments near characteristic boundaries where boundary type changes almost at random due to small numerical errors, and where customizing the boundary procedures to a transient and almost random boundary type may end up shedding unsteadiness.

\section{Acknowledgments}

I thank P. L. Roe for inspiring this work and for being a constant source of advice throughout its course. I thank A. D. French for providing the basic code for the Euler calculations.

\section{References}

1Orlanski, I., "A Simple Boundary Condition for Unbounded Hyperbolic Problems," Journal of Computational Physics, Vol, 21, 1976, pp. 251-269.
${ }^{2}$ Hedstrom, G. W., "Non-Reflecting Boundary Conditions for Nonlinear Hyperbolic Systems," Journal of Computational Physics, Vol. 30, 1979, pp. 222-237.

${ }^{3}$ Engquist, B., and Majda, A., "Absorbing Boundary Conditions for the Numerical Simulation of Waves," Mathematics of Computations, Vol. 31, 1977, pp. 629-651.

${ }^{4}$ Bayliss, A., and Turkel, E., "Radiation Boundary Conditions for Wave-Like Equations," Communications on Pure and Applied Mathematics, Vol. 33, 1980, pp. 707-725.

${ }^{5}$ Giles, M., "Non-Reflecting Boundary Conditions for Euler Equation Calculations," AIAA Paper 89-1942, 1989.

${ }^{6}$ Rudy, H., and Strikwerda, J. C., "A Non-Reflecting Outflow Boundary Condition for Subsonic Navier Stokes Calculations," Journal of Computational Physics, Vol. 36, 1980, pp. 55-70.

${ }^{7}$ Hagstrom, T., and Hariharan, S. I., "Accurate Boundary Conditions for Exterior Problems in Gas Dynamics," Mathematics of Computations, Vol. 51, 1988, pp. 581-597.

${ }^{8}$ Lindman, E. L., " 'Free-Space' Boundary Conditions for the Time Dependent Wave Equation," Journal of Computational Physics, Vol. 18, 1975, pp. 66-78.

${ }^{9}$ Higdon, R. L., "Absorbing Boundary Conditions for Difference Approximations to the Multidimensional Wave Equation," Mathematics of Computations, Vol. 47, 1986, pp. 437-459.

${ }^{10}$ Jameson, A., Schmidt, W., and Turkel, E., "Numerical Solution of the Euler Equations by Finite Volume Methods Using Runge-Kutta Time Stepping Schemes," AIAA Paper 81-1259, 1981.

${ }^{11}$ Hall, M. G., "Cell Vertex Multigrid Schemes for Solutions of the Euler Equations," Numerical Methods for Fluid Dynamics II, edited by K. W. Morton and M. J. Baines, Oxford Univ. Press, Oxford, England, UK, 1985, pp. 303-345.

${ }^{12} \mathrm{Ni}, \mathrm{R}-\mathrm{H}$., "A Multiple Grid Scheme for Solving the Euler Equations," AIAA Paper 81-1025, 1981.

${ }^{13}$ Karni, S., "Far-Field Boundaries and Their Numerical Treatment," Ph.D. Dissertation, Cranfield Inst. of Technology, Bedford, England, UK, 1990.

${ }^{14}$ Karni, S., "Far-Field Filtering Operators for the Suppression of Wave Reflections from Artificial Boundaries"' (in preparation).

${ }^{15}$ Mazaheri, K., and Roe, P. L., "New Light on Numerical Boundary Conditions,"AIAA 10th Computational Fluid Dynamics Conference, Honolulu, HI, 1991.

${ }^{16}$ Kosloff, R., and Kosloff, D., "Absorbing Boundaries for Wave Propagation Problems," Journal of Computational Physics, Vol. 63, 1986, pp. 363-374.

${ }^{17}$ Hanson, M., and Petschek, J., "A Boundary Condition for Significantly Reducing Boundary Reflections with a Lagrangian Mesh,", Journal of Computational Physics, Vol. 21, 1976, pp. 333-337.

${ }^{18}$ Israeli, M., and Orszag, S. A., "Approximation of Radiation Boundary Conditions," Journal of Computational Physics, Vol. 41, 1981, pp. 115-135.

${ }^{19}$ Roe, P. L., “Approximate Riemann Solver, Parameter Vectors, and Difference Schemes," Journal of Computational Physics, Vol. 43, 1981, pp. 357-372.

${ }^{20}$ Jespersen, D. C. . "Enthalpy Damping for the Steady Euler Equations," Applied Numerical Mathematics, Vol. 1, 1985, pp. 417-432. 